PROCEEDINGS OF THE

AMERICAN MATHEMATICAL SOCIETY

Volume 126, Number 10, October 1998, Pages 3007-3012

S 0002-9939(98)04494-3

\title{
ADDITIVITY OF QUASI-MEASURES
}

\author{
D. J. GRUBB AND TIM LABERGE
}

(Communicated by Dale E. Alspach)

\begin{abstract}
We prove that quasi-measures on compact Hausdorff spaces are countably additive. Contained in this result is a proof that every quasi-measure decomposes uniquely into a measure and a quasi-measure that has no smaller measure beneath it. We also show that it is consistent with the usual axioms of set-theory that quasi-measures on compact Hausdorff spaces are $\aleph_{1}$-additive. Finally, we construct an example that places strong restrictions on other forms of additivity.
\end{abstract}

\section{INTRODUCTION}

A quasi-measure on a compact Hausdorff space $X$ is a real-valued, non-negative set-function $\mu$ defined on $\mathcal{A}=\mathcal{A}(X)=\{A: A \subseteq X$ and $A$ is either open or closed $\}$ that satisfies the following four axioms:

1. Whenever $A \in \mathcal{A}$, then $\mu(A)+\mu(X \backslash A)=\mu(X)$.

2. If $A_{1}, A_{2} \in \mathcal{A}$ and $A_{1} \subseteq A_{2}$, then $\mu\left(A_{1}\right) \leq \mu\left(A_{2}\right)$.

3. If $A_{1}, A_{2} \in \mathcal{A}, A_{1} \cup A_{2} \in \mathcal{A}$, and $A_{1} \cap A_{2}=\emptyset$, then $\mu\left(A_{1} \cup A_{2}\right)=\mu\left(A_{1}\right)+\mu\left(A_{2}\right)$.

4. If $U \in \mathcal{A}$ is open, then $\mu(U)=\sup \{\mu(C): C$ is closed and $C \subseteq U\}$.

In general, a quasi-measure need not be sub-additive. In fact, a quasi-measure $\mu$ is the restriction of a Borel measure if and only if it is sub-additive on $\mathcal{A}$. It should also be noted that $\mathcal{A}$ is not an algebra of sets. In fact, a quasi-measure has a consistent extension to differences of closed sets if and only if it is subadditive. The proofs of these facts may be found in Wheeler's paper [W].

The first example of a quasi-measure that is not the restriction of a Borel measure was given by Aarnes in [A1]. Also in this paper is an elegant generalization of the Riesz Representation Theorem that establishes a correspondence between quasimeasures on $X$ and and functionals on $C(X)$ that are quasi-linear (i.e., linear on singly generated subalgebras). More examples and further development of the theory can be found in in [A2] and [A3].

Although quasi-measures can be defined in the completely regular case (see [B]), here we consider only quasi-measures on compact Hausdorff spaces. Accordingly, all spaces under consideration are assumed to be compact Hausdorff.

At the Workshop On Quasi-Measures held at Northern Illinois University in May 1995, Aarnes asked for an example of a quasi-measure $\mu$ on a space $X$ and a countable collection of closed subsets $\left\{C_{n}: n \in \mathbb{N}\right\}$ with $C=\bigcup_{n \in \mathbb{N}} C_{n}$ closed such that $\mu(C) \neq \sum_{n \in \mathbb{N}} \mu\left(C_{n}\right)$. The main result of this paper is to show that such

Received by the editors December 23, 1996 and, in revised form, March 13, 1997.

1991 Mathematics Subject Classification. Primary 28C15.

(C)1998 American Mathematical Society 
an example is impossible. In the course of our proof, we will also show that a quasi-measure always decomposes uniquely into the sum of a measure and a proper quasi-measure (i.e., a quasi-measure with no measure beneath it). We also show that additivity on families of cardinality $\aleph_{1}$ is consistent with the usual axioms of set theory, and construct an example that shows certain other natural types of additivity do not hold in general.

Before getting to the results of the paper, we first make some remarks on our notation. We will often speak of a set-function $\lambda$ on $\mathcal{A}$ as being a measure. By this, we mean that $\lambda$ is a sub-additive quasi-measure. By results of Wheeler $[\mathrm{W}]$, this means that $\lambda$ extends uniquely to a countably additive compact regular Borel measure on $X$, so our abuse of notation is slight.

A quasi-measure $\nu$ is proper if whenever $\lambda$ is a measure and $0 \leq \lambda \leq \nu$, then $\lambda=0$.

For $\kappa$ equal to either the first or second infinite cardinal (i.e., either $\aleph_{0}$ or $\aleph_{1}$ ), we say that a quasi-measure $\mu$ is $\kappa$-additive if whenever $\left\{A_{\alpha}: \alpha<\kappa\right\} \subseteq \mathcal{A}$ is a pairwise disjoint family and $A=\bigcup_{\alpha<\kappa} A_{\alpha} \in \mathcal{A}$, then $\mu(A)=\sum_{\alpha<\kappa} \mu\left(A_{\alpha}\right)$. If $\kappa=\aleph_{0}$, we say $\mu$ is countably additive.

If $\mu$ is a quasi-measure on $X$ and $f: X \rightarrow Y$ is continuous, then the image of $\mu$ under $f$ is the quasi-measure $\mu^{*}$ on $Y$ defined by $\mu^{*}(A)=\mu\left(f^{-1}(A)\right)$, for each $A \in \mathcal{A}(Y)$.

A subset $A$ of $X$ is solid if both it and its complement are connected. In [A3], Aarnes shows that if $X$ is connected and locally connected, then a quasi-measure is determined by its action on solid sets. We will use his notion of solid set-function (also described in [A3]) in the final section.

The support of a quasi-measure $\mu$ is the closed set $\operatorname{suppt}(\mu)=\bigcap\{K: K$ is a closed subset of $X, \mu(K)=\mu(X)$, and $\mu\lceil\mathcal{A}(K)$ is a quasi-measure $\}$. If $\operatorname{suppt}(\mu)=X$, we say $\mu$ has full support.

\section{The DECOMPOSITION THEOREM}

Our first result is of independent interest, and answers another question raised at the 1995 workshop.

Theorem 2.1 (The Decomposition Theorem). Let $\mu$ be a quasi-measure on $X$. Then there are a measure $\lambda$ and a proper quasi-measure $\nu$ such that $\mu=\lambda+\nu$. Moreover, this decomposition is unique.

Proof. Let $\mathcal{M}=\{\lambda: \lambda$ is a measure and $\lambda \leq \mu\}$. We claim that if $\lambda_{1}, \lambda_{2} \in \mathcal{M}$, then $\lambda_{1} \vee \lambda_{2} \in \mathcal{M}$. To see this, note that $\left(\lambda_{1} \vee \lambda_{2}\right)(A)=\sup \left\{\lambda_{1}\left(C_{1}\right)+\lambda_{2}\left(C_{2}\right): C_{1}\right.$ and $C_{2}$ are pairwise disjoint closed subsets of $X$ with $\left.C_{1} \cup C_{2} \subseteq A\right\} \leq \sup \left\{\mu\left(C_{1}\right)+\mu\left(C_{2}\right)\right.$ : $C_{1}$ and $C_{2}$ are pairwise disjoint closed subsets of $X$ with $\left.C_{1} \cup C_{2} \subseteq A\right\} \leq \mu(A)$.

Thus, we can define a measure $\lambda=\sup \mathcal{M}$ on $\mathcal{A}$ by $\lambda(A)=\sup \sum_{i=1}^{n} \lambda_{i}\left(C_{i}\right)$, where the supremum is taken over $\lambda_{i}$ 's in $\mathcal{M}$ and disjoint closed sets $C_{i}(i=1, \ldots, n)$ whose union is contained in $A$. By the definition of $\lambda, \lambda \leq \mu$ and the set-function $\nu=\mu-\lambda$ is a proper quasi-measure, so we are left with showing the uniqueness of the decomposition.

Suppose we have $\mu=\lambda^{\prime}+\nu^{\prime}$ where $\lambda^{\prime}$ is a measure and $\nu^{\prime}$ is a proper quasimeasure. Because $\lambda^{\prime} \in \mathcal{M}$, we have $\lambda^{\prime} \leq \lambda$. Thus, $\lambda-\lambda^{\prime}$ is a non-negative measure and $\nu^{\prime}=\left(\lambda-\lambda^{\prime}\right)+\nu$. Because $\lambda-\lambda^{\prime} \leq \nu^{\prime}$ and $\nu^{\prime}$ is proper, we have $\lambda-\lambda^{\prime}=0$. Thus, $\lambda=\lambda^{\prime}$ and $\nu=\nu^{\prime}$. 
Question 1. Does every proper quasi-measure admit a decomposition into the sum of a quasi-measure with discrete range and a quasi-measure with continuous range?

Question 2. If $\nu$ and $\nu^{\prime}$ are proper quasi-measures, is the sum $\nu+\nu^{\prime}$ also a proper quasi-measure?

\section{Countable additivity}

In addition to the decomposition theorem, we require a number of other results which are already in the literature. We state them here (mostly without proof) as lemmas for the reader's convenience.

Lemma 3.1 (The Sierpinski Theorem). Suppose $C$ is compact and connected. If $\left\{C_{n}: n \in \mathbb{N}\right\}$ is a pairwise disjoint collection of closed sets with $C=\bigcup_{n \in \mathbb{N}} C_{n}$, then there is exactly one $n \in \mathbb{N}$ such that $C_{n} \neq \emptyset$.

The next result is implicit in $[\mathrm{W}]$.

Lemma 3.2. Let $\mu$ be a proper quasi-measure on $X$ and suppose that $C \subseteq X$ is closed and 0-dimensional. Then $\mu(C)=0$.

Proof. Let $\mu$ and $C$ be as above. It is easy to see that $\mu$ restricted to the closed subsets of $C$ is a quasi-measure on $C$. Because $C$ is 0 -dimensional, this restricted quasi-measure is a measure $\lambda$ on $C$ (see [W]). But then $\lambda$ extends to a measure $\lambda^{\prime}$ on $X$ that satisfies $\lambda^{\prime} \leq \mu$. Because $\mu$ is proper, we must have $\lambda^{\prime}=0$.

The last result we need is perhaps folklore, but see the historical remarks in [E]. To set up this result, let $C \subseteq X$ be closed and let $\left\{K_{i}: i \in I\right\}$ be the decomposition of $C$ into its connected components. Define an equivalence relation $\sim$ on $X$ by identifying each $K_{i}$ to a point. More precisely, $x \sim y$ if and only if $x=y$ or $\exists i\left(x, y \in K_{i}\right)$. Let $Y=X / \sim$ be the quotient space induced by this relation and let $\pi: X \rightarrow Y$ be the induced quotient map.

Lemma 3.3. With notation as above, $\pi$ is closed (so that $Y$ is Hausdorff) and $\pi(C)$ is 0-dimensional.

We are now ready to prove our main result.

Theorem 3.4. Let $\mu$ be a quasi-measure on a compact $X$ and suppose $\left\{C_{n}: n \in \mathbb{N}\right\}$ is a pairwise disjoint collection of closed subsets of $X$ with $C=\bigcup_{n \in \mathbb{N}} C_{n}$ closed. Then $\mu(C)=\sum_{n \in \mathbb{N}} \mu\left(C_{n}\right)$.

Proof. Let $C=\bigcup_{n \in \mathbb{N}} C_{n}$ be as above. Let $\left\{K_{i}: i \in I\right\}$ be the collection of connected components of $C$. Because each $K_{i} \cap C_{n}$ is closed and $K_{i}=\bigcup_{n \in \mathbb{N}} K_{i} \cap C_{n}$, the Sierpinski Theorem implies that there is a unique $n_{i} \in \mathbb{N}$ such that $K_{i} \subseteq C_{n_{i}}$. As in Lemma 3.3, define an equivalence relation $\sim$ on $X$ by identifying each $K_{i}$ to a point. Let $Y$ be the induced quotient space and $\pi: X \rightarrow Y$ the induced quotient map.

By Lemma 3.3, $\pi$ is closed map, $Y$ is Hausdorff, and $\pi(C)$ is 0 -dimensional. Moreover, because each connected component of $C$ is contained in some $C_{n}$, we have $\pi^{-1}(\pi(C))=C, \pi^{-1}\left(\pi\left(C_{n}\right)\right)=C_{n},\left\{\pi\left(C_{n}\right): n \in \mathbb{N}\right\}$ is pairwise disjoint collection of closed subsets of $Y$, and $\pi(C)=\bigcup_{n \in \mathbb{N}} \pi\left(C_{n}\right)$.

Let $\mu^{*}$ be the image of $\mu$ under $\pi$. By the Decomposition Theorem, there are a measure $\lambda$ and a proper quasi-measure $\nu$ such that $\mu^{*}=\lambda+\nu$. By Lemma 3.2, we have each $\nu\left(\pi\left(C_{n}\right)\right)=0=\nu(\pi(C))$. Putting all of this together gives $\mu(C)=$ 
$\mu^{*}(\pi(C))=(\lambda+\nu)(\pi(C))=\lambda(\pi(C))=\lambda\left(\bigcup_{n \in \mathbb{N}} \pi\left(C_{n}\right)\right)=\sum_{n \in \mathbb{N}} \lambda\left(\pi\left(C_{n}\right)\right)=$ $\sum_{n \in \mathbb{N}} \mu^{*}\left(\pi\left(C_{n}\right)\right)=\sum_{n \in \mathbb{N}} \mu\left(C_{n}\right)$.

Corollary 3.5. Let $\mu$ be a quasi-measure on a compact Hausdorff $X$. Then $\mu$ is countably additive.

Proof. Fix a pairwise disjoint family $\left\{C_{n}: n \in \mathbb{N}\right\} \cup\left\{U_{n}: n \in \mathbb{N}\right\}$ with the $C_{n}$ 's closed, the $U_{n}$ 's open, and $A=\bigcup_{n \in \mathbb{N}} C_{n} \cup \bigcup_{n \in \mathbb{N}} U_{n} \in \mathcal{A}$.

First consider the case where $A=X$. Set $U=\bigcup_{n \in \mathbb{N}} U_{n}$ and $C=\bigcup_{n \in \mathbb{N}} C_{n}$. Notice that $C$ is closed. By a result of Aarnes in [A2], we have $\mu(U)=\sum_{n \in \mathbb{N}} \mu\left(U_{n}\right)$ and by the previous result $\mu(C)=\sum_{n \in \mathbb{N}} \mu\left(C_{n}\right)$. Thus, $\mu(X)=\mu(C)+\mu(U)=$ $\sum_{n \in \mathbb{N}} \mu\left(C_{n}\right)+\sum_{n \in \mathbb{N}} \mu\left(U_{n}\right)$.

If $A \neq X$, then $A$ is either open or closed, so we can add $X \backslash A$ to the appropriate collection of open or closed sets to obtain a collection whose union is all of $X$. The result then follows as before.

Corollary 3.6. Let $\mu$ be a 0-1 quasi-measure on a compact Hausdorff $X$ and let $C \subseteq X$ be closed. Let $\left\{K_{i}: i \in I\right\}$ be the collection of connected components of $C$. Then $\mu(C)=1$ if and only if there is an $i \in I$ such that $\mu\left(K_{i}\right)=1$.

Proof. Only the forward implication requires a proof. Suppose $C$ is closed and $\mu(C)=1$. Following the proof of Theorem 3.4, form a quotient space $Y$ by identifying each $K_{i}$ to a point. Let $\pi$ be the induced quotient map, and write $\mu^{*}=\nu+\lambda$, where $\nu$ is proper and $\lambda$ is a measure. As before, $\nu(\pi(C))=0$, so $\lambda(\pi(C))=1$. But $\lambda$ is a $0-1$ measure, hence a point-mass, so there is an $i \in I$ such that $\lambda\left(\pi\left(K_{i}\right)\right)=1$. Thus, $\mu\left(K_{i}\right)=\mu^{*}\left(\pi\left(K_{i}\right)\right)=\nu\left(\pi\left(K_{i}\right)+\lambda\left(\pi\left(K_{i}\right)\right)=1\right.$.

\section{4. $\aleph_{1}$-ADDITIVITY}

In this section, we show the statement "Every quasi-measure is $\aleph_{1}$-additive" is consistent with and independent of $Z F C$, the axioms of Zermelo-Fraenkel set theory with the axiom of choice. We will write $C H$ for the continuum hypothesis and $M A+\neg C H$ for Martin's Axiom with the negation of $C H$. Both of these statements are consistent with $Z F C$ (see $[\mathrm{K}]$ for details).

Let $M$ be a model of $Z F C+C H$. In $M$, Lebesgue measure on the unit interval fails to be $\aleph_{1}$-additive. There are also examples of proper quasi-measures which are not $\aleph_{1}$-additive in this model - consider Aarnes measure [A1] (or the example described in the next section) on the unit square.

For consistency of $\aleph_{1}$-additivity, we will need the following two consequences of $M A+\neg C H$. The first is a generalization of the Sierpinski Theorem to $\aleph_{1}$ that is due to Gruenhage [G]. It is unknown if this result can be generalized to cardinals larger than $\aleph_{1}$.

Lemma 4.1 $(M A+\neg C H)$. Suppose $C$ is compact and connected. If $\left\{C_{\alpha}: \alpha<\right.$ $\left.\aleph_{1}\right\}$ is a pairwise disjoint collection of closed sets with $C=\bigcup_{\alpha<\aleph_{1}} C_{\alpha}$, then there is exactly one $\alpha<\aleph_{1}$ such that $C_{\alpha} \neq \emptyset$.

The second is a result on additivity of Borel measures that we have paraphrased from $[\mathrm{F}]$. This lemma is actually true for all cardinals less than the continuum.

Lemma $4.2(M A+\neg C H)$. If $\lambda$ is a (compact regular Borel) measure on $X$ and $\left\{C_{\alpha}: \alpha<\aleph_{1}\right\}$ is a pairwise disjoint collection of closed subsets with $C=\bigcup_{\alpha<\aleph_{1}} C_{\alpha}$ closed, then $\lambda(C)=\sum_{\alpha<\aleph_{1}} \lambda\left(C_{\alpha}\right)$. 
Given these two results, it is easy to imitate the proof of Theorem 3.4 and its corollary to obtain the following.

Theorem $4.3(M A+\neg C H)$. If $\mu$ is a quasi-measure on a compact Hausdorff $X$, then $\mu$ is $\aleph_{1}$-additive.

Question 3. Is it consistent that quasi-measures on compact Hausdorff spaces are $\kappa$-additive for some $\kappa \geq \aleph_{2}$ ?

\section{A COUnter-example}

There are other natural forms of additivity that can be considered. In this section, $X$ will denote the unit square. In [B], it is shown that if $\mu$ is Aarnes measure on $X$ (see [A1]), then whenever $X$ is an increasing union of closed connected sets $\left\{C_{n}: n \in \mathbb{N}\right\}$, then eventually $\mu\left(C_{n}\right)=1$. In this section, we construct a counterexample that shows this form of increasing additivity fails in general.

Theorem 5.1. There is a proper 0-1 quasi-measure $\mu$ on $X$ with full support and an increasing collection $\left\{C_{n}: n \in \mathbb{N}\right\}$ of closed solid subsets of $X$ such that $X=$ $\bigcup_{n \in \mathbb{N}} C_{n}$ and each $\mu\left(C_{n}\right)=0$.

Proof. Let $Y=[1 / 4,3 / 4] \times[1 / 4,3 / 4], p_{1}=(1 / 4,1 / 4), p_{2}=(3 / 4,1 / 4)$, and $p_{3}=$ $(3 / 4,3 / 4)$. Define a solid set function $\nu$ on $\mathcal{A}(Y)$ by

$$
\nu(A)= \begin{cases}1 & \text { if }\left|A \cap\left\{p_{1}, p_{2}, p_{3}\right\}\right| \geq 2, \\ 0 & \text { otherwise. }\end{cases}
$$

Let $i: Y \rightarrow X$ be the natural embedding and let $\nu^{*}$ be the image of $\nu$ under $i$.

Let $\partial X$ denote the boundary of the unit square. Define a solid set function $\mu$ on $\mathcal{A}(X)$ by

$$
\mu(A)= \begin{cases}0 & \text { if } A \cap \partial X=\emptyset, \\ \nu^{*}(A) & \text { if } A \cap \partial X \neq \emptyset \neq(X \backslash A) \cap \partial X, \\ 1 & \text { if } \partial X \subseteq A .\end{cases}
$$

The quasi-measure determined by this solid set-function (which we also denote by $\mu$ ) is clearly a proper 0-1 quasi-measure; it can be shown that $\mu$ has full support.

Now for each $n \in \mathbb{N}$, define open sets $U_{n}$ and $V_{n}$ by

$$
U_{n}=\left(\frac{1}{2}, \frac{1}{2}+\frac{1}{n+4}\right) \times\left[0, \frac{7}{8}\right)
$$

and

$$
V_{n}=\left(\frac{1}{2}, \frac{7}{8}\right) \times\left(\frac{1}{2}, \frac{1}{2}+\frac{1}{n+4}\right) .
$$

Then set $C_{n}=X \backslash\left(U_{n} \cup V_{n}\right)$. Clearly, the $C_{n}$ 's are solid, increasing, and union up to $X$. Each $\mu\left(C_{n}\right)=0$, because $C_{n}$ splits the boundary of $X$ and $C_{n} \cap Y$ has $\nu$-measure zero $\left(C_{n} \cap Y\right.$ is the union of three closed solid $\nu$-measure zero subsets of $Y)$. 


\section{REFERENCES}

[A1] J. Aarnes. Quasi-states and quasi-measures, Adv. in Math. 86 (1991) 41-67. MR 92d:46152

[A2] J. Aarnes. Pure quasi-states and extremal quasi-measures, Math. Ann. 295 (1993) 575-588. MR 94e:46096

[A3] J. Aarnes. Construction of non-subadditive measures and discretization of Borel measures, Fund. Math. 147 (1995) 213-237. MR 96k:28022

[B] J. Boardman. Quasi-measures on completely regular spaces, Rocky Mountain J. Math., To appear.

[E] R. Engelking, General Topology, Sigma Series in Pure Mathematics, vol. 6, Heldermann, Berlin, 1989. MR 91c:54001

[F] D. Fremlin, Consequences of Martin's Axiom, Cambridge University Press, Cambridge, 1984. MR 86i:03001

[G] G. Gruenhage. Partitions of compact Hausdorff spaces, Fund. Math. 142 (1993) 89-100. MR 94a:54015

[K] K. Kunen, Set Theory, An introduction to independence proofs, Elsevier Science Publishers, Amsterdam, 1980. MR 82f:03001

[S] W. Sierpinski. Un théorème sur les continus, Tôhoku Math. J. 13 (1918) 300-303.

[W] R.F. Wheeler. Quasi-measures and dimension theory, Topology Appl. 66 (1995) 75-92. MR 96m:28002

Department of Mathematical Sciences, Northern Illinois University, Dekalb, IlliNOIS 60115

E-mail address: grubb@math.niu.edu

E-mail address: laberget@math.niu.edu 